\title{
Is the BTS/SIGN guideline confusing? A retrospective database analysis of asthma therapy
}

\section{Jordan R Covvey', Blair F Johnston', Fraser Wood², *Anne C Boyter ${ }^{1}$}

\author{
Strathclyde Institute of Pharmacy and Biomedical Sciences, University of Strathclyde, Glasgow, UK \\ ${ }^{2}$ Respiratory Consultant Physician, Forth Valley Royal Hospital, Larbert, UK
}

Originally received 12th December 2012; resubmitted 6th February 2013; revised 5th March 2013; accepted 13th March 2013; online 24th June 2013

\begin{abstract}
Background: The British guideline on the management of asthma produced by the British Thoracic Society (BTS) and the Scottish Intercollegiate Guidelines Network (SIGN) describes five steps for the management of chronic asthma. Combination therapy of a longacting $\beta_{2}$-agonist (LABA) and an inhaled corticosteroid (ICS) is recommended as first-line therapy at step 3 , although the dose of ICS at which to add a LABA is subject to debate.

Aims: To classify the inhaled therapy prescribed to patients with asthma in NHS Forth Valley according to two interpretations of the BTS/SIGN guideline and to evaluate the use of combination therapy in this population.

Methods: A retrospective analysis including patients from 46 general practitioner surgeries was conducted. Patients with physiciandiagnosed asthma were classified according to the BTS/SIGN guideline based on treatment prescribed during 2008. Patient characteristics were evaluated for the overall step classification, and specifically for therapy in step 3.

Results: 12,319 patients were included. Guideline interpretation resulted in a shift of $9.2 \%$ of patients (receiving medium-dose ICS alone) between steps 2 and 3. The largest proportion of patients (32.3\%) was classified at step 4. Age, sex, smoking status, chronic obstructive pulmonary disease co-morbidity, and utilisation of short-acting $\beta_{2}$-agonists and oral corticosteroids all correlated with step; however, no differences in these characteristics were evident between low-dose combination therapy and medium-dose ICS alone at step 3.

Conclusions: Further studies are needed to evaluate prescribing decisions in asthma. Guideline recommendations regarding the use of ICS dose escalation versus combination therapy need to be clarified relative to the published evidence.

(C) 2013 Primary Care Respiratory Society UK. All rights reserved.

JR Covvey et al. Prim Care Respir J 2013; 22(3): 290-295

http://dx.doi.org/10.4104/pcrj.2013.00060
\end{abstract}

Keywords prescribing patterns, asthma guideline, general practice, retrospective cohort

\section{Introduction}

The current British guideline for the management of asthma, which was published jointly by the British Thoracic Society (BTS) and the Scottish Intercollegiate Guidelines Network (SIGN) in 2012, is based on a five-step algorithm for the management of chronic asthma. This starts with inhaled short-acting $\beta_{2}$-agonists (SABA) for mild intermittent asthma, escalating to chronic oral steroid therapy for refractory patients. ${ }^{1}$ Early versions of the BTS guideline ${ }^{2,3}$ recommended an increase in the dose of inhaled corticosteroid (ICS) as the preferred escalation of therapy from step 2 to step 3, compared with the original collaboration published in $2003^{4}$ and the current guideline ${ }^{1}$ which now recognises the use of combination therapy of an ICS and a long-acting $\beta_{2}$-agonist (LABA) as preferred therapy at step 3. This recommendation is echoed in other international guidance produced by the Global Initiative for Asthma (GINA) ${ }^{5}$ and the National Heart, Lung and Blood Institute (NHLBI). ${ }^{6}$

This change in recommendation is underpinned by several analyses. Nearly 20 years ago Greening et al.' published an analysis comparing the use of add-on salmeterol therapy versus a 2.5-fold increase in ICS dose in patients with poorly controlled asthma prescribed a low dose $(400 \mu \mathrm{g}$ daily) of beclometasone dipropionate (BDP). Patients who received combination therapy achieved greater increases in peak expiratory flow (PEF), less diurnal variation of PEF, and reduced use of reliever inhalers than those who received a higher dose of ICS. ${ }^{7}$ Two subsequent meta-analyses ${ }^{8,9}$ confirmed the benefits of low-dose combination therapy $(400 \mu \mathrm{g}$ BDP equivalent with a LABA) compared with higher doses of ICS.

The FACET (Formoterol and Corticosteroids Establishing

\footnotetext{
* Corresponding author: Dr Anne C Boyter, Senior Lecturer, Strathclyde Institute of Pharmacy and Biomedical Sciences, University of Strathclyde, 161 Cathedral Street, Glasgow G4 ORE, UK. Tel: +44 (0)141 5484594 Fax: +44 (0)141 5522562 E-mail: anne.boyter@strath.ac.uk
} 
Therapy) group analysed the effects between varying doses of budesonide (200 vs. $800 \mu \mathrm{g}$ daily) and the addition of formoterol to either dose of ICS. ${ }^{10}$ The addition of formoterol resulted in larger improvements in lung function and asthma symptom scores, but the four-fold increase in ICS dose demonstrated a larger protective effect on mild and severe exacerbations. ${ }^{10}$ This landmark study, published after Greening et al. but before (and excluded from) the meta-analyses, supports the use of increased doses of ICS in selected patient populations despite the risk of adverse events associated with such therapy.

In view of these conflicting data, the guideline recommendation relating to the dose of ICS at which to consider combination therapy is subject to interpretation. Step 2 for adult patients recommends initiation of ICS therapy within a wide range of 200-800 $\mu \mathrm{g}$ daily of BDP equivalent, with $400 \mu \mathrm{g}$ daily considered an appropriate starting dose. ${ }^{1}$ Although step 2 specifies treatment with ICS doses up to $800 \mu \mathrm{g}$ daily, step 3 also recommends an increase in the ICS dose to $800 \mu \mathrm{g}$ daily if LABA add-on therapy is inadequate or produces no response.' Although not intended to be a stringent pathway for asthma management, the guidance from BTS/SIGN is notably less clear than that from GINA or NHLBI and may be interpreted differently by clinicians, particularly as it contains little discussion on the advantages and disadvantages of these two options.

A study by Neville et al. ${ }^{11}$ reported a classification of UK patients' asthma therapy according to the 1993 BTS guideline, ${ }^{3}$ before the preference for combination therapy was included. In this analysis, $75 \%$ of patients were prescribed step 1 or 2 therapy and fewer than $5 \%$ received low-dose combination therapy. ${ }^{11}$ With the change in the guideline recommendations since this analysis, there is a need to re-evaluate the prescribing trends in the asthma population.

The aims of this study were to stratify the therapy prescribed to patients with asthma in National Health Service (NHS) Forth Valley according to two interpretations of the BTS/SIGN guideline and to examine the use of combination therapy at step 3 in this population.

\section{Methods}

Campbell Software Solutions ${ }^{\odot}$ E-PRS clinical recording tool was implemented in 46 general practitioner (GP) surgeries in NHS Forth Valley, approximately $80 \%$ of practices in the area, during 2007 under an incentive scheme by the Scottish Government. The goal of E-PRS was to provide a disease-specific electronic template for consultation assistance and to audit clinical management. ${ }^{12}$ E-PRS was designed to interface directly with the practice administration software in place in NHS Scotland at the time, so the data are considered identical to those recorded in the patient medical record. Practice-level data from this tool have since been amalgamated for data mining at the health board level. Anonymised data for all practices involved in E-PRS were exported and stored in Microsoft SQL ${ }^{\circledR}$ Server 2008. Data were available for a two-year period and contained fields primarily related to disease management rather than outcomes.

All patients aged $>12$ years with physician-diagnosed asthma who were issued prescriptions for asthma management between 1 January 2008 and 31 December 2008 were isolated and classified according to two interpretations of the BTS/SIGN guideline, with differing ICS dose cut-offs at steps 2 and 3 (Table 1). To best assess 'real-world' practice, patients with a co-morbid diagnosis of chronic obstructive pulmonary disease (COPD) were included in the analysis and their relative effect was quantified. For the BTS/SIGN(a) interpretation, step 2 was interpreted literally and included ICS doses up to $800 \mu \mathrm{g}$ daily BDP equivalent (defined as low- or mediumdose ICS in this analysis). For the BTS/SIGN(b) interpretation, only low-dose ICS (defined as up to $400 \mu \mathrm{g}$ daily BDP equivalent) was included in step 2 and medium-dose ICS (defined as between 401 and $800 \mu \mathrm{g}$ daily BDP equivalent) was considered to be step 3 . Information on leukotriene receptor antagonists was not collected by the E-PRS tool and therefore classification of these therapies was unable to be performed in the current analysis.

Data queries were run in ascending step order with inclusion

Table 1. BTS/SIGN asthma step guideline interpretations

\begin{tabular}{|c|c|c|}
\hline Step 4 & \multicolumn{2}{|l|}{$\begin{array}{l}\text { ICS }>800 \mu g \text { (high dose) }+ \text { LABA } \\
\text { ICS }>800 \mu g \text { (high dose) } \\
\text { ICS }>800 \mu g \text { (high dose) + TP }\end{array}$} \\
\hline Step 3 & 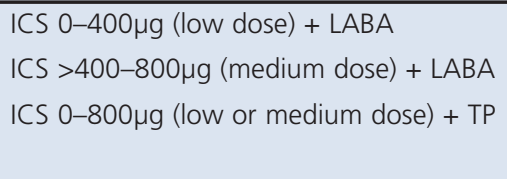 & 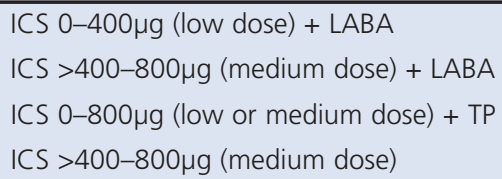 \\
\hline Step 2 & $\begin{array}{l}\text { ICS 0-400 } \mu \mathrm{g} \text { (low dose) } \\
\text { ICS }>400-800 \mu \mathrm{g} \text { (medium dose) } \\
\text { TP alone }\end{array}$ & 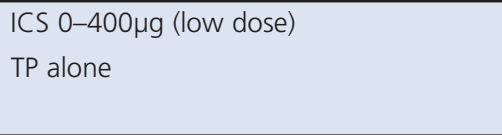 \\
\hline
\end{tabular}

BTS/SIGN=British Thoracic Society/Scottish Intercollegiate Guidelines Network, ICS=inhaled corticosteroid, LABA=long-acting $\beta$-agonist, SABA=short-acting $\beta$-agonist, TP=theophylline. 
and exclusion criteria for the therapy prescribed, designed to capture patients who were prescribed one regimen while excluding prescribing of all regimens in higher steps. The number of patients prescribed therapy at each step was determined, as well as the type of therapy prescribed. For prescriptions containing an ICS, the total daily dose was determined using the dose and frequency specified on the prescription. To account for differences in potency, doses were standardised to BDP, with budesonide considered equipotent and BDP HFA, ciclesonide, fluticasone, and mometasone twice as potent. Patients meeting the criteria for two sequential asthma steps during the year studied were assumed to have 'stepped up' and were classified into the higher step. Patients who were unable to be classified into any step were isolated and termed 'other'. Inhaled SABA therapy was permitted at all steps, as was the intermittent use of short-term oral corticosteroids (OCS), defined as a course of less than 14 days duration or with a titrating schedule.

Patient characteristics including age, sex, smoking status, COPDco-morbidity, the number of OCS courses prescribed in the previous year, and the number of SABA canisters prescribed in the previous year were assessed using descriptive statistics. The BTS/SIGN(b) interpretation was used to stratify the patients. Minitab ${ }^{\circledast} 16$ statistical software was used for analysis; t tests were used to compare for continuous variables and binomial proportion confidence intervals and $\chi^{2}$ tests were used for categorical data. A Bonferroni correction was applied to minimise error with multiple comparisons where appropriate.

Consultation with departmental ethics determined that ethics approval was not required.

\section{Results}

\section{Step classification}

A total of 12,319 patients aged $>12$ years with physician-diagnosed asthma were issued prescriptions for asthma-related medication during 2008. Eight hundred and forty-nine (6.9\%) patients had a concurrent physician diagnosis of COPD. The step classification for BTS/SIGN(a) was 1,957 (15.9\%), 3,911 (31.7\%), 1,856 (15.1\%),
3,978 (32.3\%) and 149 (1.2\%) patients for steps 1-5, respectively. Altering the step $2 / 3$ definitions to BTS/SIGN $(b)$ resulted in a shift of 1,145 patients (9.2\%) from step 2 to step 3, representing those patients who received medium-dose ICS therapy alone (Figure 1). Overall, 468 patients (3.8\%) were unable to be classified according to either guideline interpretation and were recorded as 'other'. These patients received treatment outside the guideline recommendations, including single-therapy LABA, oral SABAs, or other combinations of therapy.

The exclusion of patients with a concurrent COPD diagnosis had a small effect on the overall results, resulting in a BTS/SIGN(a) classification of 1,871 (16.3\%), 3,812 (33.2\%), 1,778 (15.5\%), $3,467(31.2 \%)$ and $112(1.0 \%)$ patients for steps $1-5$, respectively, and a shift of 1,103 (9.6\%) patients between interpretations. The proportions for steps 2 and 4 were statistically different from the original classification $(p<0.05)$.

Within step 3 of BTS/SIGN(b), medium-dose ICS alone was prescribed most frequently $(n=1,145 ; 38.1 \%)$, compared with lowdose combination therapy $(n=979 ; 32.6 \%)$ and medium-dose combination therapy $(n=806 ; 26.8 \%)$. The remaining patients $(n=73 ; 2.5 \%)$ received treatment with theophylline-containing

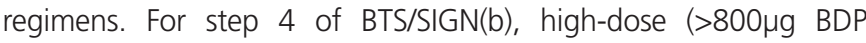
equivalent) combination therapy represented the majority of prescribing ( $n=3,035 ; 76.3 \%)$. Other therapies prescribed included high-dose ICS alone $(n=692 ; 17.4 \%)$ and theophylline-containing regimens ( $n=251 ; 6.3 \%)$.

Of the patients categorised to step 3, 158 (5.3\%) met the criteria for both steps 2 and 3, having been prescribed both low- and medium-dose ICS during the year. Similarly, 329 patients (8.3\%) categorised to step 4 met the criteria for both steps 3 and 4 and were prescribed both medium- and high-dose ICS. For prescriptions with combination therapy, a single device rather than separate inhalers was used for 6,074 patients (85.2\%).

\section{Patient characteristics}

Variation in patient characteristics was evident according to step classification (Table 2). The majority of patients were women and

Figure 1. Comparison of BTS/SIGN asthma step classification by interpretation

BTS/SIGN (a) BTS/SIGN (b)

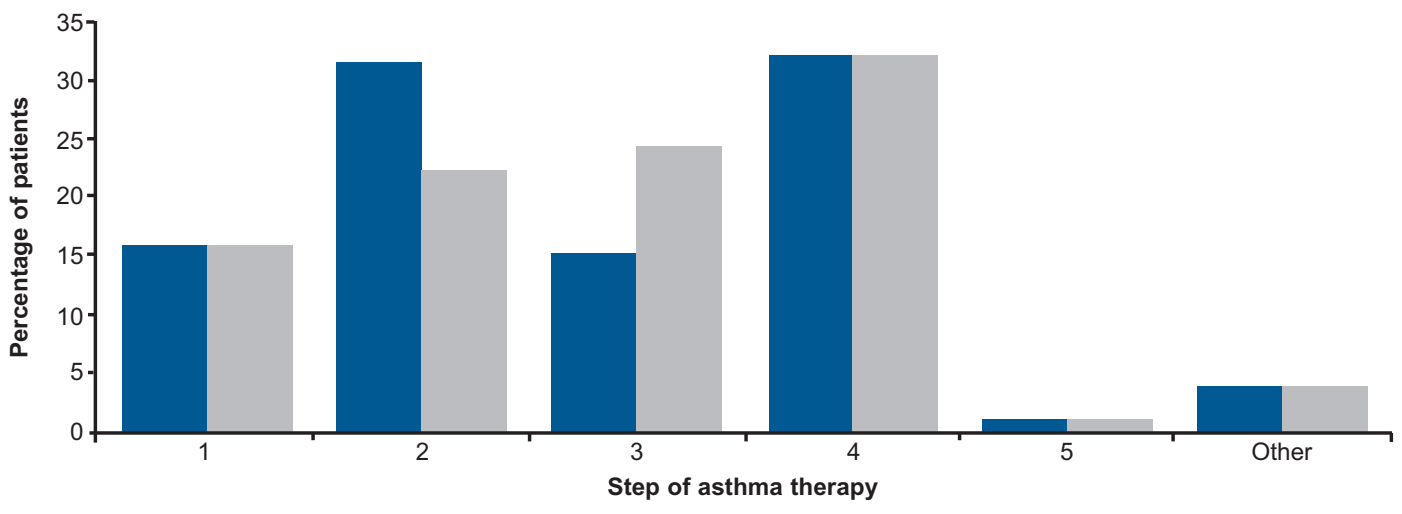




\begin{tabular}{l} 
Table 2. Patient characteristics within BTS/SIGN(b) step classification \\
\multirow{2}{*}{ Characteristics } \\
\cline { 4 - 6 }
\end{tabular}

$\mathrm{IQR}=$ interquartile range, $\mathrm{OCS}=$ oral corticosteroid, $\mathrm{SABA}=$ short-acting $\beta$-agonist, $\mathrm{SD}=$ standard deviation.

middle-aged, and the prevalence of women and median age both increased from step 1 to step 5. The prevalence of current smokers was relatively stable across all steps, ranging from $18.3 \%$ to $23.0 \%$. However, as the step increased, the number of former smokers increased from $15.7 \%$ to $36.2 \%$ while the number of never smokers decreased from $50.3 \%$ to $36.9 \%$. The prevalence of co-morbid COPD and utilisation of OCS and SABA also increased with step.

Within the treatment options in step 3, the use of OCS and SABA was similar between medium-dose ICS and low-dose combination therapy ( $p=0.067$ and $p=0.137$, respectively). Mediumdose combination therapy had higher use of OCS and SABA ( $p=0.01$ and $p=0.02$, respectively). Current tobacco use was higher for patients receiving medium-dose combination therapy $(p=0.005$; Table 3).

\section{Discussion}

\section{Main findings}

This retrospective 'snapshot' of prescribing patterns for patients diagnosed with asthma in NHS Forth Valley shows that different interpretations of the BTS/SIGN guideline resulted in a shift of $9.2 \%$ of patients between steps 2 and 3, demonstrating that a significant proportion of patients received medium-dose ICS without concurrent LABA therapy. There were no evident differences in patient characteristics for patients receiving low-dose combination therapy versus medium-dose ICS alone. Furthermore, this analysis revealed that approximately one-third of patients with asthma were treated at step 4 of the guideline.

\section{Interpretation of findings in relation to previously published work}

The literal interpretation of the guideline (BTS/SIGN(a)) allows ICS prescribing at a dose of up to $800 \mu \mathrm{g}$ BDP equivalent daily in step 2. The large proportion of patients who received a medium-dose ICS in the current analysis shows that this interpretation is likely to be prevalent, despite evidence that benefit of the addition of a LABA may be achieved at lower doses.

The risk of an exacerbation is an important aspect of asthma control. ${ }^{13}$ In addition to the work by Greening et al. ${ }^{7}$ and two metaanalyses, $^{8,9}$ a recent Cochrane review ${ }^{14}$ including 14,000 adults demonstrated the benefits of adding a LABA to a lower dose of ICS $(400 \mu \mathrm{g}$ daily BDP equivalent) compared with continued escalation of the ICS dose. In particular, the benefits were not only seen for lung function and symptom control but for also for the prevention of exacerbations. ${ }^{14}$ However, the large observational database study by Thomas et al. ${ }^{15}$ would argue, like FACET, that an increased ICS dose is more protective against exacerbations. FACET had previously found that an increase in ICS dose was associated with fewer

\section{Table 3. Selected patient characteristics within step 3 of BTS/SIGN (b)}

\begin{tabular}{l|l|l|l|l|l|l|l}
\multirow{2}{*}{ Step 3 therapy } & \multicolumn{2}{l|}{ Smoking status } & OCS & \multicolumn{2}{l|}{ SABA } \\
\cline { 2 - 8 } & Never & Current & Former & $\begin{array}{l}\text { Patients, } \\
\mathrm{n}(\%)\end{array}$ & $\begin{array}{l}\text { Mean (SD) } \\
\text { courses }\end{array}$ & $\begin{array}{l}\text { Patients, } \\
\mathrm{n}(\%)\end{array}$ & $\begin{array}{l}\text { Mean (SD) } \\
\text { canisters }\end{array}$ \\
\hline MD-ICS & $613(53.5)$ & $230(20.1)$ & $235(20.5)$ & $81(7.1)$ & $1.21(0.54)$ & $832(72.7)$ & $3.65(5.07)$ \\
\hline LD-C & $526(53.7)$ & $168(17.2)$ & $217(22.2)$ & $91(9.3)$ & $1.51(0.85)$ & $740(75.6)$ & $3.82(4.63)$ \\
\hline MD-C & $376(46.7)$ & $182(22.6)$ & $207(25.7)$ & $106(13.2)$ & $1.82(1.56)$ & $646(80.1)$ & $4.55(5.60)$ \\
\hline
\end{tabular}

LD-C=low-dose combination therapy, MD-C=medium-dose combination therapy, MD-ICS=medium-dose inhaled corticosteroid, OCS=oral corticosteroid,

$\mathrm{SABA}=$ short-acting $\beta$-agonist, SD=standard deviation. 
exacerbations, although this was demonstrated between a likely

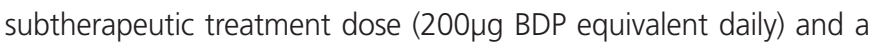
fourfold increase dose comparator $(800 \mu \mathrm{g}){ }^{10}$ while the Cochrane review comparators followed clinical stepwise management with a gradual increase in ICS dose. Although the literature remains conflicting, available evidence indicates that combination therapy is highly effective as long as the underlying dose of ICS is sufficient to control the exacerbation. ${ }^{16}$

The BTS/SIGN step scheme was not designed to be a rigid set of recommendations, as many factors may contribute to the correct therapy for a patient including inhaler technique, smoking status, and individual response variations. ${ }^{17}$ Accordingly, this analysis showed differences in patient characteristics across steps including age, smoking status, and increased use of SABA and/or OCS as markers of symptoms and exacerbations, respectively. However, our analysis found that, within step 3, the proportion and extent of patients who had previously received SABA and OCS prescriptions were similar for low-dose combination therapy and medium-dose ICS. Our results suggest that patients in whom the dose of ICS was increased did not have a greater history of exacerbations in the past year than those who were prescribed combination therapy.

The previous study based on the 1993 BTS guidelines by Neville et al. ${ }^{11}$ showed a step allocation of $27.5 \%, 47.1 \%, 6.3 \%, 10.8 \%$ and $0.8 \%$ for steps $1-5$, respectively. Our current analysis demonstrates significant increases in the proportion of patients treated at higher steps, particularly step 4. Several factors may be responsible. Three licensed combination products for asthma have been introduced since the analysis by Neville et al., reflecting the switch in prescribing guidance. Although single-agent LABA preparations have been available in the UK since 1991, the licensing of combination products is likely to have motivated prescribing changes independent of guideline recommendations. This was reflected in the wide use of single-device combination therapy in this analysis. The inclusion of patients with co-morbid COPD (disproportionately at higher steps) and the licensed use of highdose combination therapy for this indication ${ }^{18,19}$ also influenced our results; however, a repeat of our step analysis excluding these patients showed the effect to be minimal. Lastly, a high rate of smoking may have led to the use of higher doses of ICS to promote efficacy. Outside of these factors, the specific increase in prescribing of high-dose combination therapy since Neville et al. ${ }^{11}$ may be the result of more aggressive treatment of asthma over time, patient non-adherence to prescribed treatment, or hesitancy to 'step-down' asthma therapy after achieving symptom control. Although step 4 was primarily composed of high-dose combination therapy, there were also a number of patients treated with high-dose ICS alone. These patients may be the result of historical practice or the use of large increases in ICS doses during exacerbations, although this strategy is not recommended in the current guideline. ${ }^{1}$

\section{Strengths and limitations of this study}

The strengths of the current design include the large population sample and geographical diversity of the data. The NHS Forth Valley Health Board serves a population of approximately 300,000 and is home to two larger towns as well as a span of rural area, resulting in demographic diversity in the study population. As the E-PRS database collected data from the majority of practices in the Health Board, we would expect these data to be highly representative of currently treated patients with asthma. Most importantly, as this analysis stratifies patients based on prescribed treatment rather than symptom control or PEF, it provides a 'real-world' snapshot of the current management of patients with asthma in Scotland.

The study also has some limitations. The dosage for each patient (and subsequent step allocation) was determined using the instructions written on the prescription, which was available for 95\% of ICS prescriptions issued. Use outside of prescriptive instructions - such as verbal alterations from clinicians, patients varying their doses according to a self-management plan, or poor adherence - are unable to be captured by this type of retrospective analysis. This may lead to inaccuracies in the allocation of patients within the step scheme. Furthermore, we were only able to assess prescriptions issued to patients during a single calendar year, so the analysis does not take into account medication regimes trialled before this time frame and the success or failure of those interventions. While some patients might be prescribed mediumdose ICS due to previous failure of LABA therapy, this would be expected to occur infrequently. Some patients were found to have met the criteria for two steps during the study period and were assumed to have 'stepped up' in therapy. However, this was found to be the case in only a small number of the patients and demonstrates minimal movement between steps in our patients. Lastly, prescriptions for leukotriene receptor antagonists (LTRAs) were not captured by the E-PRS tool and are not included in this analysis. Pharmacy dispensing data recorded a total of 10,395 prescriptions for LTRAs dispensed in NHS Forth Valley during 2008. ${ }^{19}$ This included both adult and paediatric patients. This current analysis of adult patients included 24,023 prescriptions for ICS inhalers and 25,708 prescriptions for combination inhalers. While it is possible that inclusion of LTRAs in the step classification may have altered our final stratification, we consider this effect to be small due to the lower number of LTRA prescriptions compared with ICS/combination inhalers prescriptions, the known higher utilisation of LTRAs for paediatric patients, and the use of LTRAs primarily for the management of allergic rhinitis rather than asthma.

\section{Implications for future research, policy and practice}

The BTS/SIGN asthma guideline is an invaluable tool to guide prescribers in the treatment of asthma. While individual patient characteristics and clinician judgement are paramount in determining therapy, it is essential that guideline advice reflects current evidence and promotes good prescribing practice. It is apparent that the landscape of asthma prescribing has shifted dramatically since the publication of Neville et al., ${ }^{11}$ and that further analysis is necessary to assess clinicians' opinions of the current step management according to the guideline and to continually re-evaluate guideline recommendations. The reasons underpinning prescribing decisions at steps 3 and 4 need to be investigated for motivation and appropriateness.

\section{Conclusions}

The BTS/SIGN guideline is subject to variable interpretation. Overall, 
$15 \%$ of patients were found to be receiving medium- or high-dose ICS without adjunctive treatment with a LABA and should prompt further investigation into the clarity of guideline recommendations. Additionally, the high level of use of high-dose combination therapy should be explored further.

\section{Handling editor Hilary Pinnock \\ Statistical review Gopal Netuveli}

Acknowledgments The authors thank the NHS Forth Valley Airways Managed Clinical Network for provision of the data for this analysis.

Conflicts of interest ACB is a member of the steering group for the BTS/SIGN asthma guideline and has received honoraria from GlaxoSmithKline. JRC, BFJ and FW have no conflicts of interest.

Contributorship JRC: preparation and execution of analysis, drafted manuscript. BFJ: development of methodology, critical review of manuscript. FW: planning, critical review of the manuscript. ACB: planning and interpretation of analysis, critical review of the manuscript.

Funding JRC: PhD studentship provided in partnership by the US-UK Fulbright Commission and the University of Strathclyde.

\section{References}

1. British Thoracic Society and Scottish Intercollegiate Guidelines Network (SIGN). British guideline on the management of asthma. A national clinical guideline. (SIGN publication no. 101). http://www.sign.ac.uk/guidelines/fulltext/101/index.html (accessed 10 Sep 2012).

2. Guidelines for management of asthma in adults: $\longmapsto$ Chronic persistent asthma. Statement by the British Thoracic Society, Research Unit of the Royal College of Physicians of London, King's Fund Centre, National Asthma Campaign. BMJ 1990;301:651-3. http://dx.doi.org/10.1136/bmj.301.6753.651

3. British Thoracic Society, British Paediatric Association, Royal College of Physicians of London, et al. Guidelines on the management of asthma. Thorax 1993;48(Suppl 2):S1-24.

4. British Thoracic Society and Scottish Intercollegiate Guidelines Network. British guideline on the management of asthma. Thorax 2003;58(Suppl 1):i1-94.

5. Global Initiative for Asthma (GINA). Global strategy for asthma management and prevention. 2012. http://www. ginasthma.org/ (accessed 28 Jan 2013).

6. National Heart, Lung and Blood Institute (NHLBI). National Asthma Education and Prevention Program (NAEPP). Expert Panel Report 3 (EPR3): Guidelines for the diagnosis and management of asthma. 2007. http://www.nhlbi.nih.gov/ guidelines/asthma/asthgdln.pdf (accessed 28 Jan 2013).

7. Greening AP, Ind PW, Northfield M, Shaw G. Added salmeterol versus higher-dose corticosteroid in asthma patients with symptoms on existing inhaled corticosteroid.
Allen \& Hanburys Limited UK Study Group. Lancet 1994;344:219-24. http://dx.doi.org/10.1016/S0140-6736(94)92996-3

8. Shrewsbury S, Pyke $S$, Britton M. Meta-analysis of increased dose of inhaled steroid or addition of salmeterol in symptomatic asthma (MIASMA). BMJ 2000;320:136873. http://dx.doi.org/10.1136/bmj.320.7246.1368

9. Masoli M, Weatherall M, Holt S, Beasley R. Moderate dose inhaled corticosteroids plus salmeterol versus higher doses of inhaled corticosteroids in symptomatic asthma. Thorax 2005;60:730-4. http://dx.doi.org/10.1136/thx.2004.039180

10. Pauwels RA, Löfdahl CG, Postma DS, et al. Effect of inhaled formoterol and budesonide on exacerbations of asthma. Formoterol and Corticosteroids Establishing Therapy (FACET) International Study Group. N Engl J Med 1997;337:1405-11. http://dx.doi.org/10.1056/NEJM199711133372001

11. Neville RG, Pearson MG, Richards $\mathrm{N}$, et al. A cost analysis on the pattern of asthma prescribing in the UK. Eur Respir J 1999;14:605-09. http://dx.doi.org/10.1034/j.1399-3003.1999.14c20.x

12. Campbell Software Solutions@). E-PRS. http://www.campbellsoftware.co.uk/ products/gskcdm/ (accessed 10 Sept 2012).

13. Reddel HK, Taylor DR, Bateman ED, et al; American Thoracic Society/European Respiratory Society Task Force on Asthma Control and Exacerbations. An official American Thoracic Society/European Respiratory Society statement: asthma control and exacerbations: standardizing endpoints for clinical asthma trials and clinical practice. Am J Respir Crit Care Med 2009;180(1):59-99. http://dx.doi.org/10.1164/rccm.200801-060ST

14. Ducharme FM, Ni Chroinin M, Greenstone I, Lasserson TJ. Addition of long-acting beta2-agonists to inhaled corticosteroids versus same dose inhaled corticosteroids for chronic asthma in adults and children. Cochrane Database Syst Rev 2010; (5):CD005535. http://dx.doi.org/10.1002/14651858.CD005535.pub2

15. Thomas M, von Ziegenweidt J, Lee AJ, Price D. High-dose inhaled corticosteroids versus add-on long-acting $\beta$-agonists in asthma: an observational study. J Allergy Clin Immunol 2009;123:116-21. http://dx.doi.org/10.1016/j.jaci.2008.09.035

16. Sears MR. The addition of long-acting beta-agonists to inhaled corticosteroids in asthma. Curr Opin Pulm Med 2011;17(1):23-8. http://dx.doi.org/10.1097/MCP.0b013e328341004c

17. Haughney J, Price $D$, Kaplan A, et al. Achieving asthma control in practice: understanding the reasons for poor control. Respir Med 2008;102(12):1681-93. http://dx.doi.org/10.1016/j.rmed.2008.08.003

18. Electronic Medicines Compendium. Seretide 100, 250, 500 Accuhaler http://www.medicines.org.uk/emc/medicine/2317/SPC/ (accessed 29 Jan 2012).

19. Electronic Medicines Compendium. Symbicort Turbohaler 400/12, Inhalation powder. http://www.medicines.org.uk/emc/medicines/11882/SPC/ (accessed 29 Jan 2012).

20. NHS Scotland. Prescribing Information System for Scotland (PRISMS). http://www.isdscotland.org/Health-Topics/Prescribing-and-Medicines/PRISMS/ (accessed 15 Aug 2012).

Available online at http://www.thepcrj.org 